\title{
Transient elastography correlated to four different histological fibrosis scores in children with liver disease
}

\author{
Ulrike Teufel-Schäfer ${ }^{1,2}\left(\right.$ D $~ \cdot$ Christa Flechtenmacher $^{3} \cdot$ Alexander Fichtner $^{2} \cdot$ Georg Friedrich Hoffmann $^{2} \cdot$ \\ Jens Peter Schenk ${ }^{4}$. Guido Engelmann ${ }^{5}$
}

Received: 24 August 2020 / Revised: 15 January 2021 / Accepted: 18 February 2021 / Published online: 11 March 2021

(C) The Author(s) 2021

\begin{abstract}
Currently, liver histology is the gold standard for the detection of liver fibrosis. In recent years, new methods such as transient elastography (TE) have been introduced into clinical practice, which allow a non-invasive assessment of liver fibrosis. The aim of the present study was to investigate the predictive value of TE for higher grade fibrosis and whether there is any relevance which histologic score is used for matching. For this purpose, we compared TE with 4 different histologic scores in pediatric patients with hepatopathies. Furthermore, we also determined the aspartate aminotransferase-to-platelet ratio (APRI) score, another non-invasive method, to investigate whether it is equally informative. Therefore, liver fibrosis in 75 children was evaluated by liver biopsy, TE and laboratory values. Liver biopsies were evaluated using four common histological scoring systems (Desmet, Metavir, Ishak and Chevalier's semi-quantitative scoring system). The median age of the patients was 12.3 years. TE showed a good correlation to the degree of fibrosis severity independent of the histological scoring system used. The accuracy of the TE to distinguish between no/ minimal fibrosis and severe fibrosis/cirrhosis was good $(p=0.001$, AUC-ROCs $>0.81)$. The optimal cut-off value for the prediction of severe fibrosis was $10.6 \mathrm{kPa}$. In contrast, the APRI score in our collective showed no correlation to fibrosis.

Conclusion: TE shows a good correlation to the histological findings in children with hepatopathy, independent of the used histological scoring system.
\end{abstract}

What is Known:

- The current gold standard for detecting liver fibrosis is liver biopsy. Novel non-invasive ultrasound-based methods are introduced to clinical diagnostics.

- Most histological scores have been developed and evaluated in adult populations and for only one specific liver disease.

What is New:

- Transient elastography (TE) in children showed a good correlation to fibrosis severity irrespective of the utilized histological scoring system.

- The aspartate aminotransferase-to-platelet ratio (APRI) showed no correlation with different stages of liver fibrosis in children.

Keywords Pediatric $\cdot$ Transient elastography $\cdot$ Liver fibrosis $\cdot$ Histological scoring

Communicated by Peter de Winter

Ulrike Teufel-Schäfer

ulrike.teufel@uniklinik-freiburg.de

Christa Flechtenmacher

christa.flechtenmacher@med.uni-heidelberg.de

Alexander Fichtner

alexander.fichtner@med.uni-heidelberg.de

Georg Friedrich Hoffmann

georg.hoffmann@med.uni-heidelberg.de

Jens Peter Schenk

Jens-Peter.Schenk@med.uni-heidelberg.de

Guido Engelmann

Engelmann@lukasneuss.de
1 Department of General Pediatrics, Adolescent Medicine and Neonatology, Faculty of Medicine, Medical Center, University of Freiburg, Mathildenstr. 1, 79106 Freiburg, Germany

2 Division of Pediatric Gastroenterology and Hepatology, Center for Child and Adolescent Medicine, University Hospital Heidelberg, Im Neuenheimer Feld 430, 69120 Heidelberg, Germany

3 Department of Pathology, University Medical Centre, University of Heidelberg, Im Neuenheimer Feld 224, 69120 Heidelberg, Germany

4 Division of Pediatric Radiology, Department of Diagnostic and Interventional Radiology, University Medical Centre, University of Heidelberg, Heidelberg, Germany

5 Department of Pediatrics, Lukas Hospital, Neuss, Germany 


\section{Introduction}

Liver fibrosis and liver cirrhosis are gradual changes in the liver structure caused by chronic liver disease [1]. These structural changes usually develop slowly over years, with liver cirrhosis being the final stage of fibrotic liver disease. The clinical progression from liver fibrosis to cirrhosis may be preventable if fibrosis is detected early enough.

The current gold standard for the detection of structural changes in the liver is histological evaluation. Several histological scoring systems have been established for the classification (necroinflammatory activity) and staging (fibrosis) of liver damage [2]. However, liver biopsy also carries potential risks, especially bleeding, infection, perforation of other organs and, especially in children, the risk of sedation [3]. Sampling errors, such as sample size or puncturing away from relevant areas of the liver, can also lead to an underestimation of liver damage $[4,5]$.

Therefore, a reliable non-invasive method for the detection of liver fibrosis and liver cirrhosis is needed in clinical practice. In addition to serological tests, e.g. aspartate aminotransferaseto-platelet ratio (APRI), ultrasound-based systems for the direct or indirect determination of liver stiffness have been introduced into clinical routine. Transient elastography (TE) is based on the measurement of the velocity of a shear wave induced by a mechanical impulse in the liver. The velocity of the shear wave reflects the stiffness of the liver. The stiffness depends primarily on the amount of extracellular matrix in the liver. Therefore, liver stiffness is strongly related to the extent of fibrosis. Reference values for TE in children were published earlier by our group [6].

The aim of the present study was to investigate the predictive value of TE for higher grade fibrosis and whether there is any relevance which histologic score is used for matching. For this purpose, we compared TE with 4 different histologic scores. Furthermore, we also determined the aspartate aminotransferase-to-platelet ratio (APRI) score, another noninvasive method, to investigate whether it is equally informative.

\section{Materials and methods}

\section{Patients}

This cohort study at a tertiary center included all patients who underwent liver biopsy in our department. The anthropometric data of the patients (age, sex, weight and height) were measured at the time of biopsy. The body mass index (BMI) and the BMI standard deviation score (BMI-SDS) were calculated. The study was conducted in accordance with the principles of the Declaration of Helsinki and approved by the local ethics committee (S-510/2009). Written informed consent was obtained from all patients.

\section{Evaluation of biomarkers of hepatic fibrosis and liver enzyme testing}

In all patients, a fasting blood sample was taken on the day of the liver biopsy. Alanine aminotransferase (ALT), aspartate aminotransferase (AST), gamma-glutamyl transferase (GGT), cholinesterase (CHE), albumin, total and direct bilirubin, international normalized ratio (INR) and platelets were determined. APRI was calculated as AST/ULN $\times 100$ divided by platelets $(109 / \mathrm{L})$ according to Wai et al. [7]. The upper limit of normal (ULN) was used according to the manufacturer's recommendations. According to Wai et al., an APRI score of less than 0.5 means the absence of significant fibrosis in $85 \%$ of patients, while an APRI score above 1.5 is associated with significant fibrosis in $88 \%$ of patients.

\section{Ultrasound-based liver stiffness measurement: transient elastography}

Liver stiffness was determined with the FibroScan ${ }^{\circledR}$ (Echosens, Paris, France). The measurement was performed in all patients within 3 months before or after the liver biopsy. The measurement was performed by placing the probe in the 7th or 8 th intercostal space in the right ventral axillary line. The technique has been described in detail before [8]. Only measurements with ten valid shots, a success rate (ratio of the number of successful shots to the total number of shots) of at least $60 \%$ and an interquartile range $<0.3$ were included. The median of all successful shots was automatically calculated by the device.

TE was performed throughout by the same experienced examiner (U.T.). Depending on the thoracic circumference, different probes (M-, S-probes) were used. As recommended by the manufacturer, the M-probe $(3.5 \mathrm{mHz}$, diameter $7 \mathrm{~mm}$ ) is used for children with a thoracic circumference of more than $75 \mathrm{~cm}$. The S-probe ( $5 \mathrm{MHz}$, diameter $5 \mathrm{~mm}$ ) has two different modes (S1, S2). The S1-mode allows the examiner to take measurements in infants with a thoracic circumference of less than $45 \mathrm{~cm}$. The $\mathrm{S} 2$-mode is used for patients with a thoracic circumference of $45-75 \mathrm{~cm}$. The measurement depth is $25-65$ $\mathrm{mm}$ with the M-probe, 15-40 $\mathrm{mm}$ with S1-probe and 20-50 $\mathrm{mm}$ with the S2-probe.

\section{Liver histology}

Liver biopsies were performed using the Menghini method [9] under deep sedation with propofol. Biopsies were performed by two experienced examiners (U.T., G.E.) with a 17-gauge needle. The specimen length was $2 \mathrm{~cm}$ or longer, with at least 10 portal fields aimed at. The liver tissue was fixed in formalin 
and embedded in paraffin. The samples were stained with hematoxylin and eosin and then evaluated by an experienced hepatopathologist (C.F.). A second experienced pathologist was consulted for a double evaluation.

The following four different scoring systems were used for staging liver fibrosis:

1. Desmet score [10]: Fibrosis is qualitatively assessed on a scale of $0-4$. F0: no fibrosis, F1: portal fibrosis, F2: fibrosis with septa without disturbance of liver architecture, F3: septal fibrosis with severe disturbance of liver architecture, F4: cirrhosis.

2. METAVIR score [11] evaluates fibrosis qualitatively on a scale of 0-4. F0: absence of fibrosis, F1: portal fibrosis without septa, F2: portal fibrosis with few septa, F3: architectural distortion with numerous septa without cirrhosis, F4: cirrhosis.

3. Ishak score [12]: Fibrosis is qualitatively assessed on a scale of 0-6. F0: no fibrosis, F1: portal fibrosis of single portal fields, F2: portal fibrosis $>50 \%$ of portal fields, F3: plus single fibrotic portoportal bridges, F4: plus bridging fibrosis, F5: incomplete cirrhosis, F6: complete cirrhosis.

4. The semi-quantitative scoring system of Chevalier (SQS) [13] should be used independently of the etiology of fibrosis. It is mainly used in clinical studies and not in clinical practice. It is based on a scoring system that includes central vein, perisinusoidal fibrosis, portal field, number and size of septa.

All histological scores were further combined into a modified categorization system based on 3 levels: no/minimal fibrosis (NF), moderate fibrosis (MF) and severe fibrosis/ cirrhosis $(\mathrm{C})$, as shown in Table 1 .

\section{Statistical analysis}

IBM SPSS 20 for Windows (SPSS INC., Chicago, IL, USA) was used for statistical analyses. Unless stated otherwise, continuous variables are presented as median with range/ interquartile range (IQR) or number (percentage). Differences between groups were tested by Student's $t$-test or, if normality failed, with the Kruskal-Wallis (with Dunn's post hoc test in case of multiple testing) or Mann-Whitney $U$ rank-sum test. $p$ values $<0.05$ were regarded as statistically significant in a descriptive sense. The diagnostic performance of TE was assessed by receiver operating characteristics (ROC) curve analysis. Univariate and multivariate logistic regression analyses were performed to analyze predictors affecting TE. Variables were included in the multivariate model using a forward stepwise selection method, with a significance level of $p$ $<0.1$ for entering a variable into the model and $p \geq 0.2$ for removal of a previously selected variable. To determine the optimal cut-off values that differentiate between NF and MF in combination with $\mathrm{C}$, the Youden index method (maximum sensitivity and specificity) was used.

\section{Results}

A total of 80 patients were enrolled in the study. The indication for liver biopsy was suspected liver disease. We excluded 5 patients with juvenile hemochromatosis, bile duct atresia or acute liver failure. The characteristics of the study population are shown in Table 2 . The time interval between liver biopsy and TE ranged from the same day to 3 months (median 0 days).

\section{Liver histology and performance of TE}

According to the defined quality standards, TE was successfully performed on all patients. The procedure was fast $(170 \mathrm{~s}$, range 79-463 s), well tolerated and without complications. The median success rate was $100 \%$ (63-100\%). Liver biopsies were performed in all children without complications. In all cases, an adequate sample could be taken.

In all groups, significant differences were found when comparing the liver stiffness of the TE results of the groups no fibrosis (NF) vs cirrhosis $(C)(p=0.001)$ and the groups moderate fibrosis (MF) vs C ( $p=0.002-0.012)$. The results of TE and APRI stratification in NF, MF and C for each histological score are presented in Table 3. Between the four histological scoring systems investigated, there were no statistically significant NF-internal group differences in liver stiffness $(p=0.923)$, APRI $(p=0.976)$ and age $(p=0.953)$. Comparable results were found in the MF group (stiffness: $p$ $=0.983$, APRI: $p=0.802$, age: $p=0.961$ ) and in the $\mathrm{C}$ group (stiffness: $p=0.878$, APRI: $p=0.992$, age: $p=0.742$ ).
Table 1 Grading of fibrosis in no/ minimal fibrosis, moderate fibrosis and severe fibrosis/cirrhosis to the histological scoring

\begin{tabular}{llll}
\hline & No/minimal fibrosis & Moderate fibrosis & Severe fibrosis/cirrhosis \\
\hline Desmet & F0, F1 $(n=24 ; 32 \%)$ & F2 $(n=37 ; 49 \%)$ & F3, F4 $(n=14 ; 19 \%)$ \\
Metavir & F0, F1 $(n=25 ; 33 \%)$ & F2 $(n=35 ; 47 \%)$ & F3, F4 $(n=15 ; 20 \%)$ \\
Ishak & $0,1(n=19 ; 25 \%)$ & $2-3(n=41 ; 55 \%)$ & $4-6(n=15 ; 20 \%)$ \\
SQS & $0-3(n=22 ; 29 \%)$ & $4-11(n=42 ; 56 \%)$ & $12-17(n=11 ; 15 \%)$ \\
\hline
\end{tabular}

$S Q S$ semi-quantitative scoring system of Chevalier 
Table 2 Characteristics of included patients at the time of liver biopsy

\begin{tabular}{ll}
\hline Characteristics & Patients $(n=75)$ \\
\hline Gender (female), $n(\%)$ & $33(44 \%)$ \\
Age (years), mean (range) & $12.3(0.4-21.5)$ \\
$0-11$ years (prepubertal), $n(\%)$ & $37(49.3)$ \\
$12-18$ years, $n(\%)$ & $32(42.7)$ \\
$>18$ years, $n(\%)$ & $6(8.0)$ \\
BMI-SDS, mean (range) & $-0.01(-4.27-3.15)$ \\
Diagnoses, $n(\%)$ & \\
Autoimmune hepatitis & $14(18.7)$ \\
NASH & $10(13.3)$ \\
Wilson's disease & $8(10.7)$ \\
Liver transplantation & $8(10.7)$ \\
Metabolic liver disease & $9(12)$ \\
Hepatopathy of unknown origin & $19(25.3)$ \\
Others & $7(9.3)$ \\
ALT [U/l], mean (range) & $271(9-3884)$ \\
AST [U/1], mean (range) & $217(8-2706)$ \\
\hline
\end{tabular}

$B M I-S D S$ BMI standard deviation score, NASH non-alcoholic steatohepatitis

*Autosomal recessive polycystic kidney disease (ARPKD), PSC, hepatopathy in chronic intestinal pseudoobstruction, hepatitis B and hepatopathy in Wegener's granulomatosis

\section{Predictive performance of TE (ROC curve analysis)}

The accuracy of TE for the detection of MF and $\mathrm{C}$ was good in all histological scoring groups (AUC 0.81-0.89). Details on AUC-ROCs and $95 \%$ confidence intervals for the different histological grading scores are given in Fig. 1. Liver stiffness cut-off values (Youden index) were 10.6 kPA for Desmet, Metavir and Ishak and 17.6 kPA for SQS.

\section{Factors associated with an increased TE (regression analysis)}

In addition, we performed univariate linear regression to determine the relationship between TE and clinical indices of patients, including age, BMI-SDS, ALT, total bilirubin, albumin, CHE, INR and platelet. There was a positive correlation between total bilirubin, INR and TE. Serum albumin, CHE and platelets were negatively correlated with TE. To determine factors that are independently associated with liver fibrosis, a multivariable linear regression analysis was performed, which showed that CHE, total bilirubin and platelets were significantly associated with higher TE values (Table 4).

\section{Discussion}

The prognosis of chronic liver disease depends on the development of fibrosis and/or cirrhosis [14]. The gold standard for the definition of fibrosis stages is histology. However, most histological scores have only been developed for specific liver diseases in adult patients. For example, the Metavir score [11] has been validated in patients with hepatitis C. It is based on a nonquantitative analysis of portal and periportal fibrosis. It is also used for hepatitis B and autoimmune hepatitis, as these diseases have a similar distribution of fibrosis. In other liver diseases, fibrosis is more perivenular or perisinusoidal. Therefore, the adequacy of the Metavir score in these diseases must be questioned. The situation is similar to the Desmet and Ishak scores. In contrast, the semi-quantitative score developed by Chevallier et al. [13] includes the extent of fibrous deposits in all compartments of the liver. It can therefore be applied to any

Table 3 Results of TE in kilopascal expressed as median and IQR and APRI expressed as median and range for NF, MF and C of each histological score

\begin{tabular}{|c|c|c|c|c|c|c|c|}
\hline & $\mathrm{NF}$ & MF & $\mathrm{C}$ & $p$ value ${ }^{\#}$ & $\begin{array}{l}\text { NF vs MF } \\
\text { Post hoc* }\end{array}$ & $\mathrm{NF}$ vs $\mathrm{C}$ & MF vs C \\
\hline TE-D (kPa) & $5.75(4.8-8.9)$ & $7.1(5.8-14.5)$ & $18.65(10.4-36.3)$ & $p<0.001$ & $p=0.350$ & $p=0.0001$ & $p=0.012$ \\
\hline TE-M (kPa) & $6.1(4.8-9.1)$ & $6.8(5.8-12.7)$ & $20.0(11.4-32.4)$ & $p<0.001$ & $p=0.862$ & $p=0.0001$ & $p=0.003$ \\
\hline TE-I (kPa) & $5.3(4.6-8.1)$ & $7.1(5.8-13.2)$ & $20.0(11.4-32.4)$ & $p<0.001$ & $p=0.153$ & $p=0.0001$ & $p=0.004$ \\
\hline TE-S (kPa) & $5.75(4.8-8.8)$ & $6.95(5.8-15.3)$ & $24.9(11.4-48.0)$ & $p<0.001$ & $p=0.293$ & $p=0.0001$ & $p=0.002$ \\
\hline APRI-D & $0.54(0.19-0.99)$ & $0.73(0.45-1.44)$ & $0.78(0.4-3.01)$ & $p=0.200$ & Not applicable & & \\
\hline APRI-M & $0.57(0.19-1.25)$ & $0.65(0.44-1.17)$ & $0.8(0.42-3.08)$ & $p=0.271$ & Not applicable & & \\
\hline APRI-I & $0.44(0.19-1.05)$ & $0.65(0.45-1.17)$ & $0.8(0.42-3.08)$ & $p=0.161$ & Not applicable & & \\
\hline APRI-S & $0.47(0.18-0.87)$ & $0.73(0.45-1.77)$ & $0.8(0.42-3.08)$ & $p=0.097$ & Not applicable & & \\
\hline
\end{tabular}

$A P R I$ aspartate aminotransferase-to-platelet ratio, $C$ severe fibrosis/cirrhosis, $D$ Desmet, $I$ Ishak, $M$ Metavir, $M F$ moderate fibrosis, $n . s$. no significance, $N F$ no/minimal fibrosis, $p=p$ value, $k P a$ kilopascal, $S$ semi-quantitative scoring system of Chevalier, $T E$ transient elastography

${ }^{\#}$ Kruskal-Wallis test

*Dunn's post hoc test 


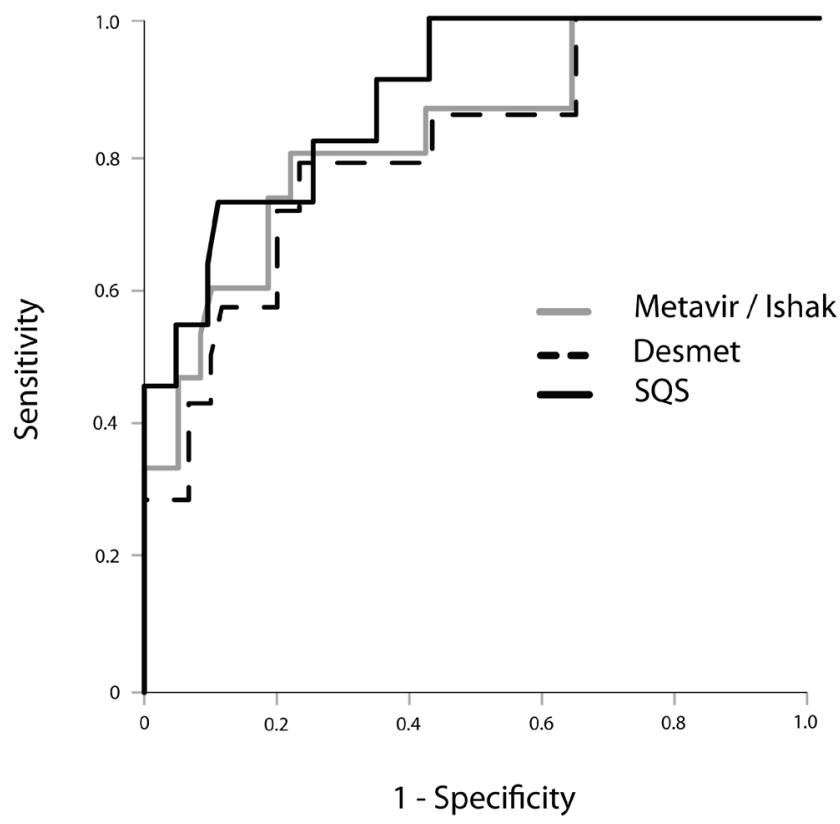

Fig. 1 ROC curve for discrimination between no/moderate fibrosis and severe fibrosis/cirrhosis by TE stratified according to different fibrosis scoring systems. Desmet-AUC $=0.81[0.68-0.94]$; Metavir/Ishak-AUC $=0.83[0.71-0.90]$; SQS-AUC $=0.89[0.80-0.98]$. AUC area under the curve, SQS semi-quantitative scoring system of Chevalier

type of chronic liver disease. However, it is rarely used in clinical routine. Although the above-mentioned scores have only been developed and evaluated in adults, they are also used in children.

Several studies have compared TE and liver biopsy in adult populations $[8,15-20]$. For example, in pediatric studies, the Metavir score has been used to assess TE in children with biliary atresia, intestinal failure, total parenteral nutrition and in children after liver transplantation [21-24]. The Ishak score has been used in pediatric populations following liver transplantation $[24,25]$ and in viral hepatitis [26]. To our knowledge, there is only one study besides Metavir in which SQS is used to evaluate

Table 4 Linear regression model to identify factors associated with increased liver stiffness

\begin{tabular}{|c|c|c|c|c|}
\hline & \multicolumn{2}{|l|}{ Univariate } & \multicolumn{2}{|l|}{ Multivariate } \\
\hline & Coefficient & $p$ value & Coefficient & $p$ value \\
\hline Age & 0.091 & 0.540 & & \\
\hline BMS-SDS & -0.427 & 0.471 & & \\
\hline ALT & 0.001 & 0.419 & & \\
\hline Bilirubin & 0.709 & $<0.001$ & 0.571 & 0.015 \\
\hline Albumin & -0.365 & 0.036 & & \\
\hline CHE & -1.792 & $<0.001$ & -1.040 & 0.033 \\
\hline INR & 23.624 & 0.001 & & \\
\hline Platelet & -0.021 & $<0.001$ & -0.021 & 0.022 \\
\hline
\end{tabular}

$B M S$-SDS body mass index standard deviation score, $A L T$ alanine aminotransferase, $C H E$ cholinesterase, INR international normalized ratio elastography [27]. In this study, 33 children underwent liver biopsy. The SQS varied between 1 and 23 (median: 10), with 11 patients having a score above 15 . TE values correlated significantly with Metavir fibrosis stages (Kendall coefficient 0.53 ; $p<0.001$ ) and SQS (Kendall coefficient $0.49 ; p<0.001$ ). To date, no pediatric study has been published that evaluates the TE value and uses the Desmet score.

Furthermore, there is no consensus on a histological scoring system for the classification of childhood liver fibrosis that is adapted to the different types of liver diseases in children. Therefore, we investigated TE in correlation with four widely used histological scores including a semi-quantitative test. Our results show that TE has a good correlation to each of the four systems. The accuracy of TE for differentiation between NF and C was good, with AUC-ROCs of more than 0.81 in severe fibrosis/cirrhosis.

The median liver stiffness increased gradually depending on the degree of fibrosis. It had no influence on which histological score was used. However, the TE could not distinguish between different low degrees of fibrosis. Of course, it would be advantageous if even minor stages of fibrosis could be distinguished by TE. Thus, depending on the underlying disease, further progression of fibrosis to cirrhosis could be avoided by adjusting the therapy.

With regard to the differentiation of the different stages of fibrosis, the results of the published data are contradictory: Behairy et al. [26], who used the Ishak score, reported a good, significant differentiation of earlier fibrosis stages, while Friedrich-Rust et al. [28] and de Ledinghen et al. [27], who used the Metavir score, only reported a good performance in differentiating between cirrhosis and low-grade fibrosis, but not between different low grades of fibrosis. One reason could be the wider range of the Ishak score (7 stages) compared to the Metavir score (5 stages) or our aggregation (3 stages).

The optimal cut-off value for the diagnosis of cirrhosis was $10.6 \mathrm{kPa}$ for Metavir, Desmet and Ishak and $17.6 \mathrm{kPa}$ for SQS. This is comparable with data from adult studies [29]. So far, there are few studies that deal with cut-off values in childhood. Ledighen et al. [27] describe in their study a comparable cutoff of $9.9+/-0.6 \mathrm{kPa}$ for various liver diseases. However, only $33(28 \%)$ of 116 children received a liver biopsy. In his cohort, the distribution in the fibrosis groups was very different. None had F0, and 54\% had an F3-F4 Metavir score. In an Italian study with 52 children with advanced fibrosis, a cut-off of 10.2 kPA was found [30]. Compared to our study, Nobili included children from the age of 4 years, the cohort size was smaller and all children had non-alcoholic steatohepatitis. There is a study by Lee et al. [31] with a similarly heterogeneous study group as ours and comparable cohort size. However, patients with cholestasis were not excluded here. Lee et al. [31] give an optimal cut-off of $8.6 \mathrm{kPa}$ to distinguish advanced fibrosis from low fibrosis. The lower value could be due to the fact that $25 \%$ of the TE measurements in this group were 
performed more than 6 months after the liver biopsy. In our cohort, the time interval between liver biopsy and TE ranged from the same day to 3 months (median 0 days).

Good accuracy of simple non-invasive tests such as the APRI score for the prediction of liver fibrosis and cirrhosis in various primary liver diseases has been demonstrated in several studies with adults [7, 32, 33]. In this study, we could not detect significant differences of the APRI between the histologically defined severity grades (fibrosis, cirrhosis) (Table 3). Hence, the predictive performance was low. There are controversial results in pediatric studies. De Ledinghen [27] and Kim [34] showed a significant correlation of the APRI score with histology in children with chronic liver disease, while Hukkinen [35] and Diaz [22] described the detection of cirrhosis by APRI but could not show a difference between the low stages of fibrosis. Interestingly, de Ledinghen [27] found a good correlation between APRI and Metavir stages, but not between APRI and SQS scores. Lind et al. [36] examined 31 children with biliary atresia. The APRI scores showed no difference between the Metavir categories. Our results are quite similar. The APRI score in our setting of different hepatopathies did not distinguish cirrhosis from grade $0 / 1$ fibrosis. We could show a slight increase in APRI score with increasing fibrosis, but no significant correlation between APRI and the severity of fibrosis. There may be several reasons for this phenomenon. Changes in platelet count and AST develop late in the course of the disease, and AST may even be normal in patients with compensated cirrhosis. Another reason could be the upper limit of normality of AST, which is age-related and depends on the manufacturer's recommendation [37]. To date, there are no international manufacturer-independent AST values.

To determine factors that are independently associated with liver fibrosis, we performed multivariate linear regression analysis. As a result, CHE, total bilirubin and platelets were independent variables associated with higher TE. Shan et al. [38] also described platelet count as a relevant factor. In their study, 181 adults with various liver diseases were included. Other relevant determinants were albumin, BMI and prothrombin activity. The liver enzymes had no correlation with fibrosis. However, these data are inconsistent. Tapper et al. [39] showed a correlation between increased ALT values and liver stiffness. However, these statistical phenomena do not help in the diagnosis of severe fibrosis or cirrhosis.

The present study has some limitations. One of the main limitations is the sample size with relatively small numbers, especially in the group with severe fibrosis/cirrhosis. In addition, our study cohort includes a heterogeneous group of liver diseases, although this group of diseases is quite representative for the hepatopathies in children that are also found in clinical practice.

TE is not proposed as an alternative to biopsy for diagnostic purposes. However, TE can be a useful tool for quickly and easily gaining an initial impression of structural changes in the liver. It also allows easy follow-up of already known structural changes in the liver. Therefore, a validated non-invasive marker for fibrosis could help to decide which patients need a biopsy to diagnose the underlying liver disease and could reduce the number of liver biopsies in children with chronic liver disease. In this study, we could show that there is a good correlation between staging of fibrosis and the results of TE regardless of the histological scoring system used. However, a significant distinction could not be shown at lower fibrosis stages. We could not demonstrate any significant differences of the APRI between the histologically defined severity grades in our very heterogeneous pediatric study population.

Abbreviations ALT, Alanine aminotransferase; APRI, Aspartate aminotransferase-to-platelet ratio; ARPKD, Autosomal recessive polycystic kidney disease; AST, Aspartate aminotransferase; BMI, Body mass index; BMI-SDS, Body mass index standard deviation score; CHE , Cholinesterase; GGT , Gamma-glutamyl transferase; INR , International normalized ratio; IQR , Interquartile range; PSC, Primary sclerosing cholangitis; ROC, Receiver operating characteristics; SD , Standard deviation; SQS , Semi-quantitative scoring system of Chevalier; TE, Transient elastography; ULN, Upper limit of normal

Code availability N/A

Authors' contributions U.T. designed the study, collected data, performed liver biopsies and transient elastography measurements. She drafted the initial manuscript and wrote the final manuscript as submitted and undertook a part of statistical analysis. C.F. examined liver specimens and classified them into different scores. A.F. undertook the statistical analysis. G.F.H. critically reviewed and revised the final manuscript as submitted. J.P.S. performed peri-interventional ultrasound and revised the final manuscript as submitted. G.E. co-designed the study, performed liver biopsies and revised the final manuscript as submitted.

Funding Open Access funding enabled and organized by Projekt DEAL. This study was funded by the Dietmar Hopp Foundation. The funds went to the Children's Hospital in Heidelberg and not to a specific person.

Data availability Can be provided upon request.

\section{Declarations}

Ethics approval The study was conducted in accordance with the principles of the Declaration of Helsinki and approved by the local ethics committee (S-510/2009).

Consent to participate Informed consent was obtained from all individual participants included in the study.

Consent for publication Patients signed a consent form for the publication of the data collected. We have no photos or personalized data in the publication.

Conflict of interest No conflict of interest: C.F., A.F., G.F.H., J.P.S. and G.E. U.T.S. reports lecture fee from AbbVie, outside the submitted work.

Open Access This article is licensed under a Creative Commons Attribution 4.0 International License, which permits use, sharing, 
adaptation, distribution and reproduction in any medium or format, as long as you give appropriate credit to the original author(s) and the source, provide a link to the Creative Commons licence, and indicate if changes were made. The images or other third party material in this article are included in the article's Creative Commons licence, unless indicated otherwise in a credit line to the material. If material is not included in the article's Creative Commons licence and your intended use is not permitted by statutory regulation or exceeds the permitted use, you will need to obtain permission directly from the copyright holder. To view a copy of this licence, visit http://creativecommons.org/licenses/by/4.0/.

\section{References}

1. Berenguer M, Schuppan D (2013) Progression of liver fibrosis in post-transplant hepatitis C: mechanisms, assessment and treatment. J Hepatol 58:1028-1041

2. Standish RA, Cholongitas E, Dhillon A, Burroughs AK, Dhillon AP (2006) An appraisal of the histopathological assessment of liver fibrosis. Gut 55:569-578

3. Westheim BH, Østensen AB, Aagenæs I, Sanengen T, Almaas R (2012) Evaluation of risk factors for bleeding after liver biopsy in children. J Pediatr Gastroenterol Nutr 55:82-87

4. Colloredo G, Guido M, Sonzogni A, Leandro G (2003) Impact of liver biopsy size on histological evaluation of chronic viral hepatitis: the smaller the sample, the milder the disease. J Hepatol 39: 239-244

5. Maharaj B, Maharaj RJ, Leary WP, Cooppan RM, Naran AD, Pirie D, Pudifin DJ (1986) Sampling variability and its influence on the diagnostic yield of percutaneous needle biopsy of the liver. Lancet 8:523-525

6. Engelmann G, Gebhardt C, Wenning D, Wühl E, Hoffmann GF, Selmi B, Grulich-Henn J, Schenk JP, Teufel U (2012) Feasibility study and control values of transient elastography in healthy children. Eur J Pediatr 171:353-360

7. Wai CT, Greenson JK, Fontana RJ, Kalbfleisch JD, Marrero JA, Conjeevaram HS, Lok ASF (2003) A simple noninvasive index can predict both significant fibrosis and cirrhosis in patients with chronic hepatitis C. Hepatology 38:518-526

8. Sandrin L, Fourquet B, Hasquenoph JM, Yon S, Fournier C, Mal F, Christidis C, Ziol M, Poulet B, Kazemi F, Beaugrand M, Palau R (2003) Transient elastography: a new noninvasive method for assessment of hepatic fibrosis. Ultrasound Med Biol 29:1705-1713

9. Menghini G (1958) One-second needle biopsy of the liver. Gastroenterology 35:190-199

10. Desmet VJ, Gerber M, Hoofnagle JH, Manns M, Scheuer PJ (1994) Classification of chronic hepatitis: diagnosis, grading and staging. Hepatology 19:1513-1520

11. Bedossa P, Poynard T (1996) An algorithm for the grading of activity in chronic hepatitis C. The METAVIR Cooperative Study Group. Hepatology 24:289-293

12. Ishak K, Baptista A, Bianchi L, Callea F, De Groote J, Gudat F, Denk H, Desmet V, Korb G, MacSween RN et al (1995) Histological grading and staging of chronic hepatitis. J Hepatol 22:696-699

13. Chevallier M, Guerret S, Chossegros P, Grimaud JA (1994) A histological semiquantitative scoring system for evaluation of hepatic fibrosis in needle liver biopsy specimens: comparison with morphometric studies. Hepatology 20:349-355

14. Schuppan D, Afdhal NH (2008) Liver cirrhosis. Lancet 371:838851

15. Castéra L, Vergniol J, Foucher J, Le Bail B, Chanteloup E, Haaser M, Darriet M, Couzigou P, De Ledinghen V (2005) Prospective comparison of transient elastography, Fibrotest, APRI, and liver biopsy for the assessment of fibrosis in chronic hepatitis C. Gastroenterology 128:343-350

16. Frulio N, Trillaud H (2013) Ultrasound elastography in liver. Diagn Interv Imaging 94:515-534

17. Yeshua H, Oren R (2008) Non invasive assessment of liver fibrosis. Ann Transplant 13:5-11

18. Ziol M, Handra-Luca A, Kettaneh A, Christidis C, Mal F, Kazemi F, de Lédinghen V, Marcellin P, Dhumeaux D, Trinchet JC (2005) Noninvasive assessment of liver fibrosis by measurement of stiffness in patients with chronic hepatitis C. Hepatology 41:48-54

19. Fung J, Lai CL, Chan SC, But D, Seto WK, Cheng C, Wong DK, Lo CM, Fan ST, Yuen MF (2010) Correlation of liver stiffness and histological features in healthy persons and in patients with occult hepatitis B, chronic active hepatitis B, or hepatitis B cirrhosis. Am J Gastroenterol 105:1116-1122

20. de Lédinghen V, Douvin C, Kettaneh A, Ziol M, Roulot D, Marcellin P, Dhumeaux D, Beaugrand M (2006) Diagnosis of hepatic fibrosis and cirrhosis by transient elastography in HIV/ hepatitis C virus-coinfected patients. J Acquir Immune Defic Syndr 41:175-179

21. Shin NY, Kim MJ, Lee MJ, Han SJ, Koh H, Namgung R, Park YN (2014) Transient elastography and sonography for prediction of liver fibrosis in infants with biliary atresia. J Ultrasound Med 33: 853-864

22. Díaz JJ, Gura KM, Roda J, Perez-Atayde AR, Duggan C, Jaksic T, Lo CW (2013) Aspartate aminotransferase to platelet ratio index correlates with hepatic cirrhosis but not with fibrosis in pediatric patients with intestinal failure. J Pediatr Gastroenterol Nutr 57:367371

23. Mutanen A, Lohi J, Heikkilä P, Koivusalo AI, Rintala RJ, Pakarinen MP (2013) Persistent abnormal liver fibrosis after weaning off parenteral nutrition in pediatric intestinal failure. Hepatology 58:729-738

24. Venturi C, Sempoux C, Bueno J, Pinas JCF, Bourdeaux C, AbarcaQuinones J, Rahier J, Reding R (2012) Novel histologic scoring system for long-term allograft fibrosis after liver transplantation in children. Am J Transplant 12:2986-2996

25. Goldschmidt I, Stieghorst H, Munteanu M, Poynard T, Schlue J, Streckenbach C, Baumann U (2013) The use of transient elastography and non-invasive serum markers of fibrosis in pediatric liver transplant recipients. Pediatr Transplant 17:525-534

26. Behairy B-S, Sira MM, Zalata KR, Salama e-SE, Abd-Allah MA (2016) Transient elastography compared to liver biopsy and morphometry for predicting fibrosis in pediatric chronic liver disease: does etiology matter? World J Gastroenterol 22:4238-4249

27. de Lédinghen V, Le Bail B, Rebouissoux L, Fournier C, Foucher J, Miette V, Castera L, Sandrin L, Merrouche W, Lavrand F et al (2007) Liver stiffness measurement in children using FibroScan: feasibility study and comparison with Fibrotest, aspartate transaminase to platelets ratio index, and liver biopsy. J Pediatr Gastroenterol Nutr 45:443-450

28. Friedrich-Rust M, Ong MF, Martens S, Sarrazin C, Bojunga J, Zeuzem S, Herrmann E (2008) Performance of transient elastography for the staging of liver fibrosis: a meta-analysis. Gastroenterology. 134:960-974

29. Wong GL (2013) Update of liver fibrosis and steatosis with transient elastography (Fibroscan). Gastroenterol Rep (Oxf) 1:19-26

30. Nobili V, Vizzutti F, Arena U, Abraldes JG, Marra F, Pietrobattista A, Fruhwirth R, Marcellini M, Pinzani M (2008) Accuracy and reproducibility of transient elastography for the diagnosis of fibrosis in pediatric nonalcoholic steatohepatitis. Hepatology 48:442-448

31. Lee CK, Perez-Atayde AR, Mitchell PD, Raza R, Afdhal NH, Jonas MM (2013) Serum biomarkers and transient elastography as predictors of advanced liver fibrosis in a United States cohort: the Boston children's hospital experience. J Pediatr 163:1058-1064 
32. Halfon P, Bacq Y, De Muret A, Penaranda G, Bourliere M, Ouzan D, Tran A, Botta D, Renou C, Brechot MC et al (2007) Comparison of test performance profile for blood tests of liver fibrosis in chronic hepatitis C. J Hepatol 46:395-402

33. Yang HR, Kim HR, Kim MJ, Ko JS, Seo JK (2012) Noninvasive parameters and hepatic fibrosis scores in children with nonalcoholic fatty liver disease. World J Gastroenterol 18:1525-1530

34. Kim SY, Seok JY, Han SJ, Koh H (2010) Assessment of liver fibrosis and cirrhosis by aspartate aminotransferase-to-platelet ratio index in children with biliary atresia. J Pediatr Gastroenterol Nutr 51:198-202

35. Hukkinen M, Kivisaari R, Lohi J, Heikkilä P, Mutanen A, MerrasSalmio L, Pakarinen M (2016) Transient elastography and aspartate aminotransferase to platelet ratio predict liver injury in paediatric intestinal failure. Liver Int 36:361-369

36. Lind RC, Verkade HJ, Porte RJ, Hulscher JBF (2012) Aspartate transaminase-to-platelet ratio index is not correlated with severity of fibrosis or survival in children with biliary atresia. J Pediatr Gastroenterol Nutr 54:698

37. Neuschwander-Tetri BA, Ünalp A, Creer MH (2004) The upper limits of normal for serum ALT levels reported by clinical laboratories depend on local reference populations. Arch Intern Med 168: 663-666

38. Shan R, Yin H, Yang W, Li J, Thang M, Zhao M, Shao J, Wang A (2016) Influencing factors of transient elastography in detecting liver stiffness. Exp Ther Med 12:2302-2306

39. Tapper EB, Cohen EB, Patel K, Bacon B, Gordon S, Lawitz E, Nelson D, Nasser IA, Challies I, Afdhal N (2012) Levels of alanine aminotransferase confound use of transient elastography to diagnose fibrosis in patients with chronic hepatitis $\mathrm{C}$ virus infection. Clin Gastroenterol Hepatol 10:932-7.e1

Publisher's note Springer Nature remains neutral with regard to jurisdictional claims in published maps and institutional affiliations. 